\title{
HIGH SENSITIVITY MEMS ULTRASOUND ARRAYS BY LATERAL FERROELECTRIC POLARIZATION
}

\author{
J.J. Bernstein , J. Bottari, K. Houston, G. Kirkos, and R. Miller \\ The Charles Stark Draper Laboratory, \\ Cambridge MA 02139-3563
}

\author{
B. Xu, Y. Ye and L.E. Cross \\ Materials Research Lab, Penn State University, \\ University Park, PA 16802
}

\begin{abstract}
This paper discusses the design of advanced micromachined ferroelectric ultrasound transducers for use at $3 \mathrm{MHz} .16 \times 16$ arrays of resonant monomorph sensors have been constructed, with sol-gel PZT as the active ferroelectric layer deposited on insulating layers of $\mathrm{ZrO}_{2}$ and $\mathrm{SiO}_{2}$. A novel in-plane polarization of the PZT is used to maximize sensitivity, while trading off reduced output capacitance to match the CMOS buffer electronics. This results in about $30 \mathrm{~dB}$ improved sensitivity compared to conventional polarizing across the thickness of the PZT layer. Fluid-filled through wafer holes are used as an acoustic matching network to achieve resonance at both 1 and 3 MHz. A lumped element equivalent circuit model is presented, as well as Finite Element Analysis results. Performance predictions for projector efficiency and receive response are given. Test results are presented including transmit response, receive sensitivity, and frequency response.
\end{abstract}

\section{INTRODUCTION}

There is great interest in two-dimensional arrays of acoustic transducers for 3-D acoustic imaging. Previous work reported the use of micromachining technology combined with sol-gel PZT (Lead Zirconate Titanate) to create 2-dimensional arrays of $\mathrm{MHz}$ acoustic transducers on silicon wafers [1]. Micromachined Ultrasonic Transducers (MUT's) based on electrostatic transducers have been developed for both air and underwater applications [2]. Miniature ultrasonic transducers have been fabricated on silicon using films of PVDF (Poly-vinylidenedifluoride) as the transduction material $[3,4]$.

In this paper we describe a novel in-plane polarization technique for increasing the sensitivity of ferroelectric monomorphs by $30 \mathrm{~dB}$ or more. Potential applications of these transducer arrays include a hand-held diver's sonar, medical ultrasound imaging, and nondestructive testing (NDT).

\section{THICKNESS MODE VS. IN-PLANE POLARIZATION}

Ferroelectric films on silicon are usually grown on an inert conducting layer such as $\mathrm{Pt}$ which forms one of the device electrodes, with the other electrode on the upper surface. Most work with PZT on silicon has been confined to thin films less than $1 \mu \mathrm{m}$ thick of PZT, although films as thick as $12 \mu \mathrm{m}$ can be deposited [5]. For a uniaxial stress $\sigma_{x x}$ in a PZT film of thickness $t_{P Z T}$ (Fig. 2) the output voltage is

$$
V=\sigma_{x x} \cdot t_{P Z T} \cdot g_{31}
$$

where $g 31$ is the receive coefficient of the PZT for a stress applied orthogonal to the poling direction.
Fig. 2 shows the normal thickness-mode polarization of a piezoelectric or ferroelectric film. For a ferroelectric film, the possibility exists to pole the film in the plane of the substrate (Fig. 3), so that much larger inter-electrode $l$ spacing is possible. The output voltage for this case is

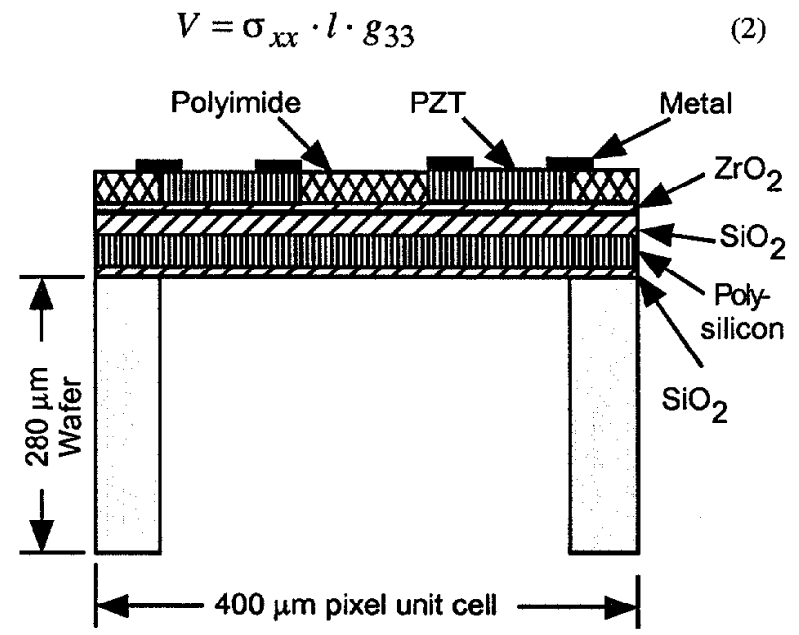

Fig. 1. Cross-section of one sensing pixel showing fluid-filled hole terminating on sensing diaphragm.

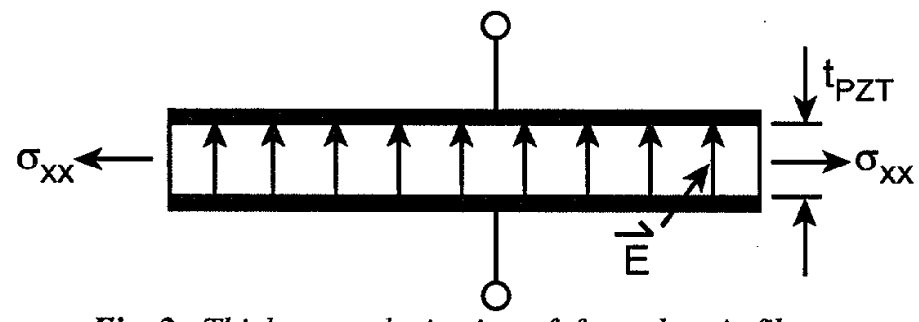

Fig. 2. Thickness polarization of ferroelectric film.

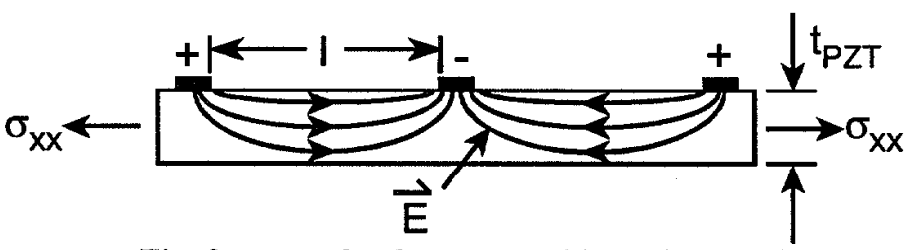

Fig. 3. Lateral polarization of ferroelectric film.

Since $g_{33}$ is normally twice $g_{31}$, a $6 \mathrm{~dB}$ increase in coupling coefficient results from using in-plane polarization. If we assume a 1 $\mu \mathrm{m}$ thick PZT film with 30 micron electrode spacing, then one obtains a factor of 30 increase in voltage sensitivity from electrode spacing, and a factor of 2 from higher ferroelectric coupling constant, for a total of $60 \mathrm{X}$ higher sensitivity $(+35.5 \mathrm{~dB})$. An additional benefit is that only a single conductive layer is required, which greatly simplifies the fabrication process. This increase in sensitivity is 
obtained at the cost of reduced output capacitance, but an overall increase in signal/noise is achieved.

Poling Procedure: The PZT films are poled using the transducer's electrodes at 10 volts/ $\mu \mathrm{m}$ of inter-electrode gap [6]. Over-voltage of $20 \mathrm{~V} / \mu \mathrm{m}$ causes the PZT film to crack. $16 \mathrm{X} 16$ arrays were poled one row at a time using a probe card with 16 high voltage electrodes and 1 ground electrode. The poling voltage was applied slowly using a ramp up, soak, and ramp down of 2 minutes each.

\section{Sensitivity-Capacitance Tradeoff}

For simple ferroelectric pressure transducers, the quantity $\mathrm{M}_{0}{ }^{2} \mathrm{C}$ (where $\mathrm{M}_{0}$ is the open-circuit sensitivity (V/Pa) and $\mathrm{C}$ is device capacitance) is characteristic of the transducer and is not affected by partitioning the transducer into series or parallel connected pieces, attaching an ideal transformer, or reconfiguring the electrodes. Hence capacitance can be traded off for sensitivity. The optimal signal to noise ratio for a capacitive sensor is achieved when the sensor capacitance is approximately equal to the input capacitance of the buffer amplifier.

The sonar pixels were designed to have an output capacitance of between 0.2 and $0.6 \mathrm{pF}$, to match the input capacitance of the CMOS readout circuit, which was $0.25 \mathrm{pF}$.

\section{ANALYTICAL MODEL}

A lumped element acoustic model (Fig. 4) of the transducer was analyzed to provide insight into the design and optimization of the transducer. The model contains an acoustic source in the far-field, acoustic radiation elements $L_{R}$ and $R_{R}$ representing radiation mass and resistance [7], a transmission line $T L$ which represents wave propagation down the fluid-filled holes, and

lumped impedances $C_{\text {dia }}$ and $L_{\text {dia }}$ which represent the compliance and mass of the air-backed diaphragm.

This lumped element model can be solved analytically, allowing optimization to be carried out. In addition the model provides insights which are absent from the finite element results.

Maximum sensitivity occurs at the system resonances, when the reactances cancel leaving only the radiation resistance.

The thickncss of the wafer was chosen to provide resonance at both $1 \mathrm{MHz}$ and $3 \mathrm{MHz}$, the desired operating frequencies of an underwater sonar. This calculated to a wafer thickness of approximately $295 \mu \mathrm{m}$ which was used for fabrication.

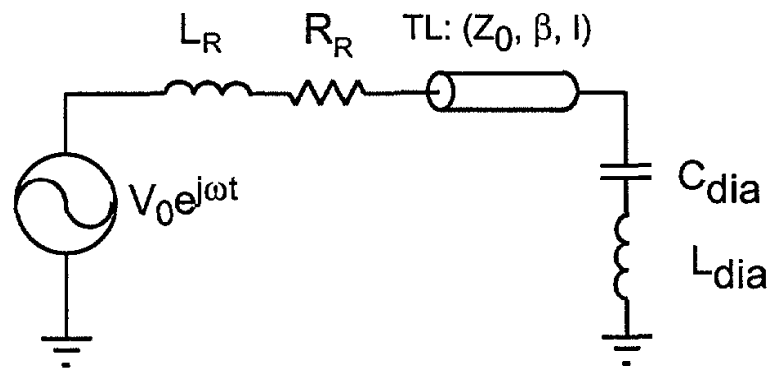

Figure 4. Lumped element equivalent circuit model of monomorph pixel on a fluid-filled hole.

Fig. 5 shows the calculated average diaphragm deflection (per $\mathbf{P a}$ input) vs. frequency for 3 wafer thicknesses which give the first, second or third resonance at $3 \mathrm{MHz}$. To achieve the first resonance at $3 \mathrm{MHz}$ required a wafer thickness of only $43 \mu \mathrm{m}$, which is too thin to handle. To achieve the third resonance at 3
$\mathrm{MHz}$ required a wafer $540 \mu \mathrm{m}$ thick. but would not yield a peak near $1 \mathrm{MHz}$ as desired. A $4^{\text {th }}$ (diagonal) line is added for reference which shows the free particle displacement of a $1 \mathrm{~Pa}$ acoustic wave in water.

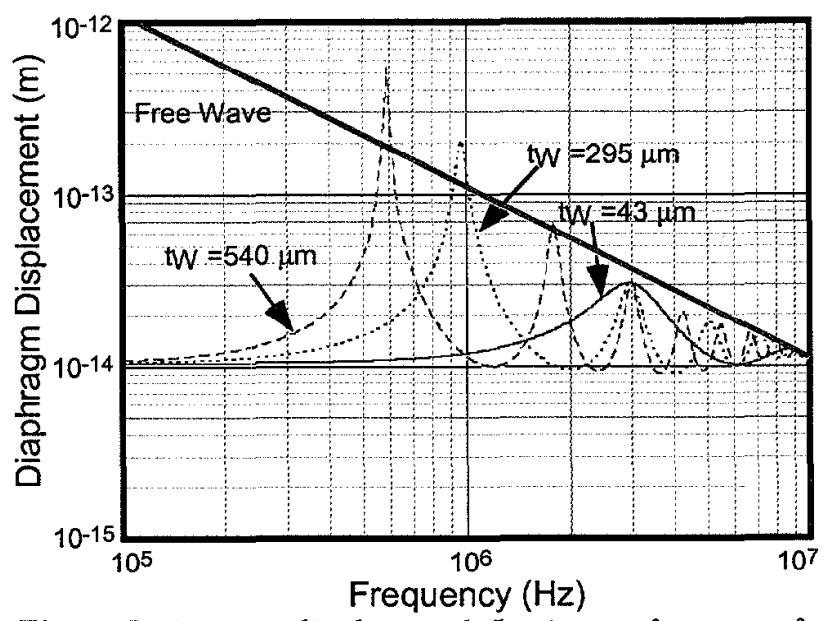

Figure 5. Average diaphragm deflection vs. frequency for sensor diaphragm mounted on hole with three different lengths to achieve a resonance at $3 \mathrm{MHz}$

\section{FINITE ELEMENT ANALYSIS}

Finite element analysis was carried out on 13 different final designs. All pixels measure $0.4 \mathrm{~mm} \mathrm{X} 0.4 \mathrm{~mm}$. The designs are grouped into 3 general types: Quad designs (Fig. 6) with 4 diaphragms per unit cell, "racetrack" designs with 2 or 3 diaphragms per unit cell (Fig. 7), and Nona designs with 9 diaphragms / unit cell.

Fig. 6 shows a quad cell, with 4 diaphragms wired in series. The center metal bond pad is for bump bonding to a CMOS "TransmitReceive Integrated Circuit" developed by Lockheed Martin IRIS.

ANSYS was used for all FEA computations. Fig. 8 shows the device as simulated by ANSYS. The diaphragm model contains the details of all layers, and was electrically driven by $1 \mathrm{~V}$.

The PZT response to the driving voltage deforms the diaphragm and generates an outgoing acoustic wave. The semi-infinite fluid filled space is represented by a hemisphere of fluid elements (the "mushroom cap" of Fig. 8). The outer boundary of this hemisphere is made up of "infinite absorbing elements" which prevent reflections back towards the transducer. .

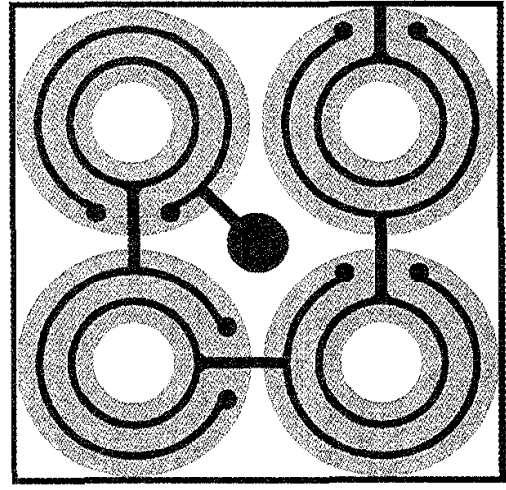

Fig. 6. Quad transducer has 4 diaphragms wired in series per unit cell $(0.4 \mathrm{~mm} \times 0.4 \mathrm{~mm})$. Concentric electrodes and PZT layer are shown. 


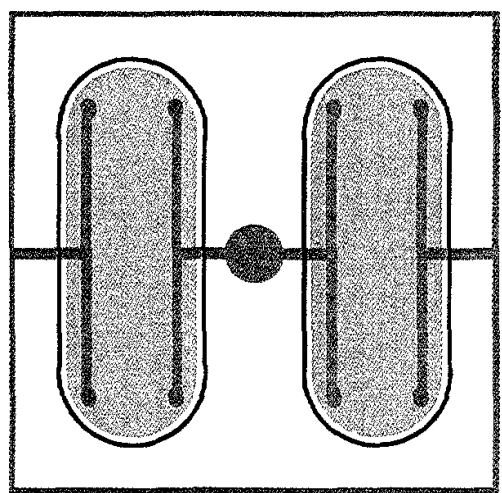

Fig. 7. "Race-track" transducer has two elongated diaphragms wired in parallel per unit cell.

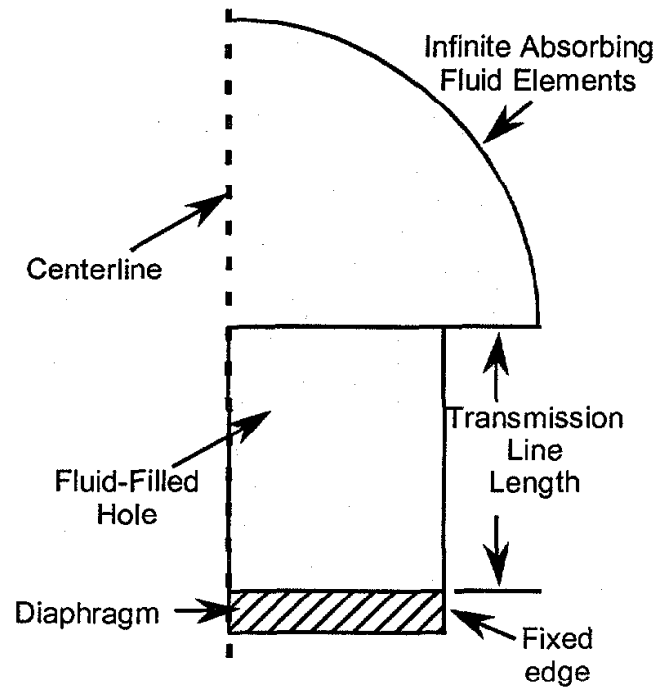

Fig. 8. FEA problem as solved with ANSYS for radially symmetric diaphragm (centerline shown) with diaphragm fixed at outer edge.

The transmit response for the transducer at 1 meter was calculated assuming conservation of energy in the acoustic wave and expressed in standard sonar units as $\mathrm{dB}$ ref. $1 \mu \mathrm{Pa} / \mathrm{V}$ at one meter.

Two approaches were used to predict receive sensitivity: static calculations augmented by the $\mathrm{Q}$ of the transducer, and reciprocity relations which allow the receive sensitivity to be directly calculated from the transmit response for any reciprocal transducer.

\section{Predicted Transmit and Receive Sensitivity}

The FEA results were translated into standard sonar units for transmit and receive. These are listed for several transducer designs in Table 1 below. Receive sensitivity was calculated from transmit response using reciprocity relations.

\section{DEVICE FABRICATION}

Sonar transducer arrays were fabricated on $100 \mathrm{~mm}$ diameter silicon wafers $290 \mu \mathrm{m}$ thick. After initial oxidation, a $3 \mu \mathrm{m}$ thick layer of undoped polysilicon was deposited by Low Pressure Chemical Vapor Deposition at $570^{\circ} \mathrm{C}$ from silane, followed by deposition of $2 \mu \mathrm{m}$ of $\mathrm{SiO}_{2}$. The wafers were then coated with $0.5 \mu \mathrm{m}$ of $\mathrm{ZrO}_{2}$ and then $3 \mu \mathrm{m}$ of PZT using sol-gel techniques
[6]. The PZT layer was patterned using conventional photolithography and wet etching.

Table 1. Predicted Transmit and Receive Responses

\begin{tabular}{|c|c|c|c|}
\hline Transducer & Unit Cell & Transmit resp. & Receive \\
\hline Name & Cap. & 1 Unit Cell & Sensitivity \\
\hline Units $\rightarrow->$ & (F) & dB// $\mu \mathrm{Pa} / \mathrm{V} @ 1 \mathrm{~m}$ & $\mathrm{~dB} / \mathrm{N} / \mu \mathrm{Pa}$ \\
\hline quad80 & $2.21 \mathrm{E}-13$ & 114.1 & -209.0 \\
\hline nona50 & $6.64 \mathrm{E}-13$ & 120.4 & -213.5 \\
\hline RT120 & $3.42 \mathrm{E}-13$ & 114.1 & -211.2 \\
\hline
\end{tabular}

A $3 \mu \mathrm{m}$ polyimide dielectric layer is then spun on and cured. This low dielectric constant layer reduces the stray capacitance to the substrate of all metal lines which are not in contact with the PZT. A metal layer consisting of Ti/Pt/Au is then sputtered and patterned by a standard liftoff technique. The wafers are then coated with a top-coat of polyimide and patterned to expose the bondpads only.

Holes are then etched from the back of the chip using an STS Inductively Coupled Plasma etcher, masked with a thick photoresist layer. This etcher allows vertical holes to be etched through the wafer.

"Mono" devices with a single diaphragm per pixel are shown in Fig. 9. This device had an output capacitance of $0.7 \mathrm{pF}$ and sensitivity $(-210 \mathrm{~dB} / / 1 \mathrm{~V} / \mu \mathrm{Pa})$.

Fig. 10 shows a cross-section of an array which has been cracked to reveal the hole side-walls. The etch walls are close to vertical.

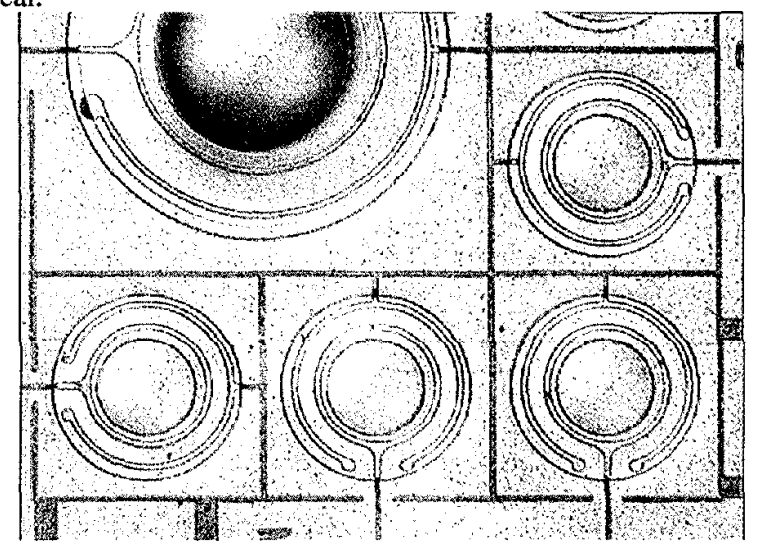

Fig. 9. Circular "Mono" pixels are poled radially with concentric ring electrodes.

\section{TEST RESULTS}

$16 \times 16$ arrays were tested at Lockheed Martin by bumpbonding an electronics readout chip to the array. In-house testing was carried out by mounting small $3 \times 3$ arrays into Kovar flat-packs with holes cut in the package to allow water and acoustic signals to reach the chip. The chips were wire-bonded to the flat-pack, which was soldered to a PC board, to which coax cables were attached. The entire assembly was then coated with silicone rubber for waterproofing, with a hole to allow sound to reach the sensor array.

Fig. 11 shows receive voltage sensitivity for a Mono-150 device (single diaphragm, radius $=150 \mu \mathrm{m}$ ). Peak response of $-210 \mathrm{~dB}$ ref. $\mathrm{V} / \mu \mathrm{Pa}$ is at $3.8 \mathrm{MHz}$, (slightly higher than the desired system frequency), with an additional peak at $1.5 \mathrm{MHz}$. Noise floor of the system is also shown. This data was compensated for $190 \mathrm{pF}$ of stray cable capacitance and pre-amp input impedance on each pixel. 


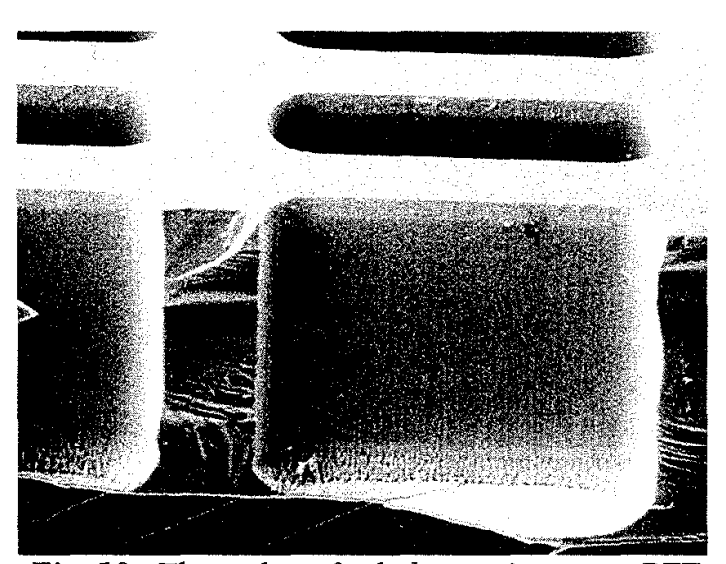

Fig. 10. Through-wafer hole terminates on PZT monomorph diaphragm.

Fig. 12 shows transmit response from a Mono-150 design pixel. The peak transmit response (at $3.8 \mathrm{MHz}$ ) is about $122 \mathrm{~dB} / /$ $\mu \mathrm{Pa} / \mathrm{V}$ at 1 meter. A peak at $1.5 \mathrm{MHz}$ is also visible.

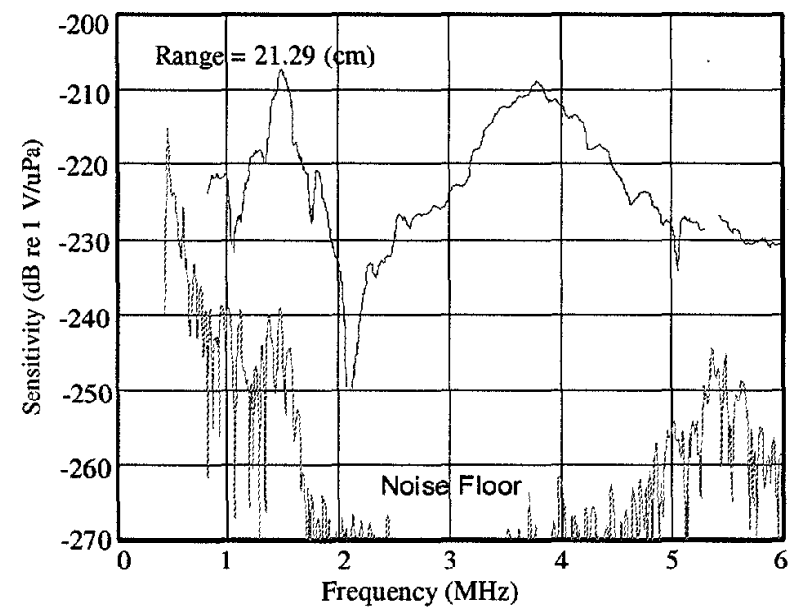

Fig. 11. Receive data for Mono 150 design pixel. Notice broad peak at $3.8 \mathrm{MHz}$.

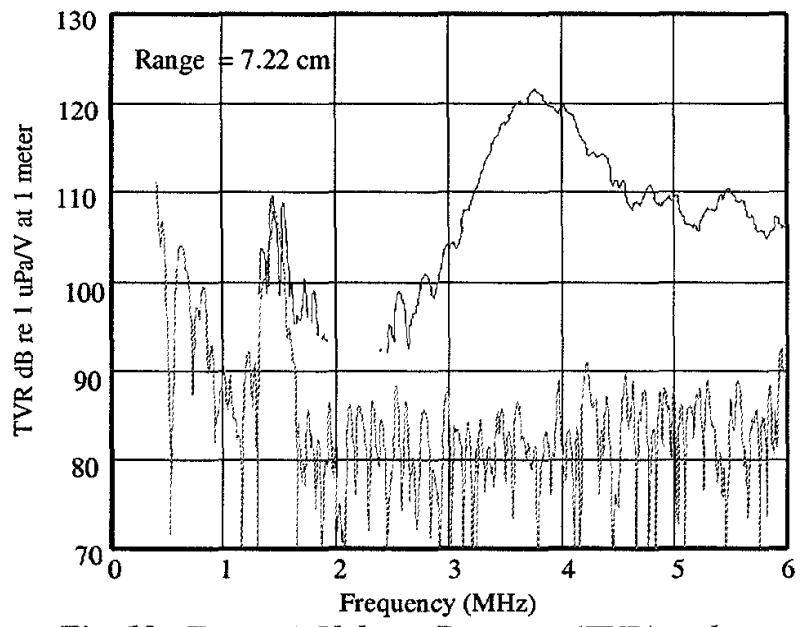

Fig. 12. Transmit Voltage Response (TVR) and noise floor, MONO 150 pixel on a test chip.

\section{SUMMARY AND CONCLUSIONS}

This paper has described a new type of MEMS ferroelectric transducer, the monomorph with in-plane polarization. This device allows sensitivity to be traded off against capacitance to optimize the system signal/noise ratio, particularly with low input-capacitance CMOS amplifiers. A novel dielectric buffer layer $\left(\mathrm{ZrO}_{2}\right)$ compatible with PZT sol-gel deposition was developed. $16 \times 16$ arrays of MEMS sonar pixels were successfully built and tested. Sensitivity of $-210 \mathrm{~dB}$ ref. $1 \mathrm{~V} / \mu \mathrm{Pa}$ at $3 \mathrm{MHz}$ was attained, which is an improvement of about $30 \mathrm{~dB}$ over previous MEMS ferroelectric sensors at a lower frequency $(0.8 \mathrm{MHz})[1]$. The devices are reciprocal and can be used for both transmit and receive.

\section{ACKNOWLEDGEMENTS}

The authors gratefully acknowledge support from the DARPA Sonoelectronics program as well as expert program monitoring from Bruce Johnson at NAVEODTECHDIV. I would also like to acknowledge the contributions of M.C. Cardoso in chip fabrication, J.O. Miller in chip poling and testing, and J. Paglia for program management.

\section{REFERENCES}

1. J. Bernstein, S.L. Finberg, K. Houston, L.C. Niles, H.D. Chen, L.E. Cross, K.K. Li, and K. Udayakumar., "Micromachined High Frequency Ferroelectric Sonar Transducers", IEEE Transactions on Ultrasonics, Ferroelectrics and Frequency Control, Vol. 44, No. 5, September 1997, pp. 960-969.

2. X. Jin, I. Ladabaum, and B.T. Khuri-Yakub, "The Microfabrication of Capacitive Ultrasonic Transducers", IEEE Journal of Microelectromechanical Systems, Vol. 7, \# 3, September 1998, pp. 295-302.

3. R.G. Swartz, Application of Polyvinylidene Fluoride to Monolithic Silicon PVF2 Transducer Arrays, Ph.D. Dissertation, Stanford University, (May 1979).

4. J.H. Mo, J.B. Fowlkes, A.L. Robinson, and P.L. Carson, "Crosstalk Reduction with a Micromachined Diaphragm Structure for Integrated Ultrasound Transducer Arrays," IEEE Trans. Ultrasonics, Ferroelectrics and Frequency Control, Vol. 38, (January 1992), pp. 48-53.

5. H.D. Chen, K.R. Udayakumar, C.J. Gaskey, L.E. Cross, J.J. Bernstein, and L.C. Niles, "Fabrication and electrical properties of lead zirconate titanate thick films", Journal of the American Ceramic Society, vol. 79, no.8, pp. 2189-92, 1996.

6. B. Xu, Y. Ye, L.E. Cross, J. Bernstein and R. Miller, "Dielectric hysteresis from transverse electric fields in lead zirconate titanate thin films", Applied Physics Letters, vol. 74, \# 23, pp. 3549-3551, (7 June 1999).

7. Kinsler, Frey, Coppens and Sanders, Fundamentals of Acoustics, $3^{\text {rd }}$ edition, (John Wiley and Sons, 1982), pp. 191-193.

8. Leo L. Beranek, Acoustical Measurements, 1988 Edition, (Acoustical Society of America / American Institute of Physics, 1988), pp. 113-122.

9. R. J. Bobber, Underwater Electro Acoustic Measurements, (Peninsula Press, 1988 ) pp. 27-45. 